\title{
LA ALFABETIZACIÓN DE LOS FUTUROS DOCENTES EN INVESTIGACIÓN EDUCATIVA: UNA REFLEXIÓN TEÓRICA DESDE EL CONTEXTO DE CHILE
}

\section{The Literacy of Future Teachers in Educational Research: A Theoretical Reflection from the Context of Chile}

\author{
Haylen Perines* \\ Universidad de La Serena, Chile \\ \} \text { haylen.perines@userena.cl } \\ ID https://orcid.org/0000-0002-7020-1014
}

\author{
Karla Campaña \\ Universidad de La Serena, Chile \\ 、kcampana@userena.cl \\ ID http://orcid.org/0000-0001-6074-4448
}

\begin{abstract}
Resumen
El presente artículo busca describir cómo se puede alfabetizar a los futuros docentes en investigación educativa con la mirada puesta en Chile y Latinoamérica, asumiendo que su formación académica de pregrado carece de un sistema intencionado para educarlos al respecto. En la revisión teórica, se describen cuatro elementos que deben ser considerados por los actores educativos involucrados en el desarrollo de la carrera docente: 1) la presencia de la investigación en los programas y en el perfil de egreso de las carreras de pedagogía; 2) la importancia de que los formadores de profesores tengan un rol investigador activo; 3) la utilización de los artículos de investigación como recurso pedagógico; 4) la incorporación de estudiantes de pedagogía en proyectos de investigación, asumiendo que es fundamental que los docentes del mañana desarrollen gradualmente un habitus investigativo que contribuya a su mejor preparación profesional. Como principales hallazgos destacamos la idea defendida por Catalán (2017) en cuanto alfabetizar a los futuros docentes implica la compleja tarea de comprender que como educadores no solo deben ser receptores o consumidores de conocimiento, sino que también deben participar en su producción. El artículo finaliza con algunas reflexiones a modo de conclusión que enfatizan los cambios urgentes y las profundas transformaciones que necesita la formación docente en materia de investigación. Palabras clave: formación docente, docentes, investigación educativa, universidad, curriculum.
\end{abstract}

\begin{abstract}
This article seeks to describe how future teachers in educational research can be literate with the focus on Chile and Latin America and assuming that their pre-grade academic training lacks an intentional system to educate them about it. In the theoretical review, four elements are described that should be considered by the educational actors involved in the development of the teaching career: the presence of the research in the programs and in the profile of exit of the careers of pedagogy, the Importance of teacher trainers having an active investigative role, the use of research articles as a pedagogical resource
\end{abstract}

ISSN (impreso): 2636-2139

* Autora de correspondencia.

ISSN (en línea): 2636-2147

Sitio web: https://revistas.isfodosu.edu.do/recie

Recibido: 14 de marzo de 2019

Aprobado: 20 de mayo de 2019

\section{COMO CITAR:}

Perines, H., \& Campaña, K. (2019). La alfabetización de los futuros docentes en investigación educativa: Una reflexión teórica desde el contexto de Chile. Revista Caribeña de Investigación Educativa (RECIE), 3(1), 7-18. https://doi.org/10.32541/recie.2019.v3i1.pp7-18 
and the incorporation of students of pedagogy in research projects, assuming that it is essential that Tomorrow's teachers will gradually develop a research habit that contributes to their best professional preparation. As main findings, we highlight the idea defended by Catalan, (2017) as literate to future teachers implies the complex task of understanding that as educators should not only be receivers or consumers of knowledge but also must Participate in producing it. The article ends with some reflections by way of conclusion, which emphasize the urgent changes and the profound transformations that the teaching training needs in research subjects.

Keywords: teacher training, teachers, educational research, university, curriculum.

\section{Introducción}

"Conocemos muy poco, casi nada, solo hay una asignatura lo cual no es suficiente para formar a una persona en el tema". "Si se formara mejor a los docentes, la solución a los problemas educativos sería más profesional”. Estas palabras no son extraídas de ninguna teoría, sino que son parte del testimonio de los futuros profesores espańoles que participaron en el estudio de Perines y Murillo (2017a) palabras que, sin duda, despiertan interés y también preocupación.

Estas opiniones se complementan con las valoraciones de algunos docentes en otros estudios: la investigación no sirve para nada; tiene una mala reputación; los artículos son demasiado abstractos y los temas que se investigan interesan exclusivamente a los investigadores, entre otros (p. e., Barte1s, 2003; Kaestle, 1993; Gore \& Gitlin, 2004; Murillo \& Perines, 2017; Perines, 2016; Perines \& Murillo, 2017a, Perines \& Murillo, 2017b; Vanderlinde \& van Braak, 2010).

En estos trabajos se reitera una conclusión: la formación de los docentes juega un rol fundamental para que éstos se involucren activamente en la investigación. Lograr cambios en este sentido es un desafío para los formadores de profesores y para el funcionamiento institucional de las universidades. Pero, ¿por qué es importante que los futuros profesores se involucren en la investigación y aprendan de ella? "Formar profesionales que utilicen los métodos de la ciencia para transformar la realidad es una necesidad”, afirman Rubio, Torrado, Quirós y Valls (2018, p.336), enfatizando la importancia del tema en la formación profesional pedagógica.

En tal sentido, la generación y el uso del conocimiento son primordiales. Es fundamental que los futuros profesores se conviertan en profesionales reflexivos y no sólo en emisores mecánicos del currículum (Larenas, Rodríguez, Hernández, Solar \& Morales, 2015).

Que un profesor sea reflexivo favorece la calidad de la docencia, pues le permitirá replantearse sus propias ideas y conocimientos y tomar decisiones informadas y autónomas (Scherping, 2010). En ese sentido la investigación educativa es crucial. Resulta curioso que -aunque la formación en investigación sea indicada por la evidencia empírica como un tema prioritario en el que se debe trabajar-, existen pocos trabajos que profundicen en ello. Si bien hay estudios centrados en cómo los profesores en ejercicio se relacionan con la investigación educativa (Anwaruddin, 2016; Hall, Leat, Wall, Higgins \& Edwards, 2006; Haberfellner \& Fenzl, 2017; Pekel \& Akçay, 2018; Procter, 2013; Simons, Kushner, Jones \& James, 2003; Taylor, 2013), todavía faltan más investigaciones sobre cómo se da esta dinámica con los docentes en formación.

Con estos antecedentes, el objetivo de este artículo es describir cómo se puede alfabetizar a los futuros docentes en investigación educativa en Chile, entendiéndola como una "indagación sistemática y mantenida, planificada y autocrítica, que se halla sometida a crítica pública y a las com- 
probaciones empíricas en donde estas resulten adecuadas" (Stenhouse, 1987, p.35). Nos situamos en Chile porque es el escenario de interés de las autoras de este escrito y porque en dicho país se están llevando a cabo cambios importantes en el currículo de las pedagogías, en cuanto a una renovación curricular con modificaciones del perfil de egreso, situando al egresado como un profesional competente frente a las demandas de la escuela. Tales cambios hacen aún más relevante este análisis.

Señalamos que el término "alfabetización de los profesores en investigación" no ha surgido espontáneamente en la redacción de este trabajo, sino que es una expresión ya mencionada por otros autores. En un estudio realizado con profesores en ejercicio, Anwaruddin y Pervin (2015) concluyen que los docentes no se comprometen con la investigación porque no han sido "alfabetizados" en ello, pues no contaron con esa educación en sus universidades ni tampoco en la formación permanente que reciben una vez ingresan al mundo laboral. La pregunta es: ¿cómo lograr esta alfabetización?

\subsection{La presencia de la investigación en los programas y en el perfil de egreso de las carreras de pedagogía en Chile}

En general, cada institución educativa centra su trabajo en la capacitación de profesores de acuerdo con las habilidades que han establecido como relevantes y en coherencia con su perfil de egreso (Grossman \& McDonald, 2008; Slavin, 2002). Así, la incorporación de la investigación no se establece como una obligatoriedad en la preparación profesional, por lo que el lugar que ocupa en el currículo depende de las decisiones internas de cada universidad (Harris \& Sass, 2011).

La formación de profesores en Chile está orientada por los estándares pedagógicos y disciplinares, los cuales no aluden directamente a una formación en investigación educativa, sin embargo, plantean que el recién egresado, sea cual sea la pedagogía en la que fue formado, debe poseer "conocimientos de análisis cuantitativo que le permitan leer, analizar e interpretar los distintos tipos de datos" (CPEIP, 2012, p.16).

Así mismo, en algunos planes de estudio de pedagogía existen solo una o dos asignaturas relacionadas directamente con la investigación, que forman parte de la línea de Licenciatura en Educación, las cuales, además, se llevan a cabo en los últimos semestres de la carrera y que forman parte de la línea de Licenciatura en Educación (ver tabla 1). 
Tabla 1:

Asignaturas relacionadas con investigación educativa en programas de pedagogía básica de universidades estatales chilenas

\begin{tabular}{|l|l|c|}
\multicolumn{1}{|c|}{ Universidad Estatal } & \multicolumn{1}{c|}{$\begin{array}{c}\text { Nombre de Asignatura } \\
\text { Universidad de Tarapacá }\end{array}$} & $\begin{array}{c}\text { Semestre en que se } \\
\text { imparte }\end{array}$ \\
\hline Universidad de Atacama & Investigación de la Acción Educativa & VI \\
\hline Universidad de La Serena & Metodología de la Investigación Científica & V de la Investigación Educacional \\
\hline Universidad de Chile & Taller de Reflexión e Investigación (identidad docente) & VII \\
\hline Universidad Santiago de Chile & Metodología de la Investigación & II \\
\hline Universidad O’Higgins & Investigación y Reflexión Pedagógica I & III \\
\hline Universidad del Bío Bío & Investigación y Reflexión Pedagógica II & VI \\
\hline & Investigación Educacional Cuantitativa & VII \\
\hline & Investigación Educacional Cualitativa & V \\
\hline
\end{tabular}

Nota: La revisión se ha realizado sobre la base de la oferta de las universidades estatales, presentes en sus sitios web durante el mes de abril del año 2018. Fuente: Elaboración propia.

Para lograr una alfabetización de los futuros profesores, esta incorporación tan puntual y escasa de la investigación debe cambiar. Un docente que conoce los fundamentos de la investigación desde el inicio de su formación puede aprender a utilizar los estudios científicos de una forma constructiva para su profesión (Rust, 2009), pudiendo acceder a nuevos conocimientos sobre su propia práctica (Counsell, Evans, McIntyre \& Raffan, 2000).

La incorporación de la investigación en sus programaciones curriculares requiere que las instituciones que forman a los docentes, y sus respectivos líderes, establezcan políticas internas de trabajo que incluyan explícitamente la investigación educativa entre sus lineamientos (Burn, 2007; Lampert, 2009). Ahora bien, es pertinente establecer qué tipo de investigación es la más adecuada en el escenario de la formación de profesores. Es por ello que, desde la perspectiva de este trabajo, la investigación que tenga repercusiones en la formación docente debe ser capaz de complementar los paradigmas cuantitativos, cualitativos y socio-críticos, y hacerlos inteligibles a los estudiantes. El énfasis de una alfabetización no debe estar en que los futuros profesores manejen a la perfección uno u otro enfoque epistemológico, sino en la orientación que el docente proporciona al estudiante, para que éste sea capaz de reflexionar sobre el contenido de un determinado texto científico, evaluar sus implicaciones en el contexto pedagógico y llevar a cabo pequeńas investigaciones pertinentes a su nivel académico.

En el camino hacia estos cambios están sucediendo cosas importantes, como la renovación curricular de las carreras de pedagogía en algunas universidades públicas chilenas, entre ellas la Universidad de La Serena (ULS). La ULS ha iniciado un proceso de implementación del currículum renovado en sus once carreras de pedagogía, en las cuales se optimiza la presencia y gradualidad de las asignaturas que tienen relación con la investigación en comparación con el currículum antiguo. El primer cambio que queda a la vista es que todas las asignaturas que desarrollan elementos de la investigación educativa tienen un orden secuencial y cada una es pre-requisito de la siguiente. Asi- 
mismo, se cambian parcialmente las asignaturas del plan antiguo y se agrega una más, para un total de 5 asignaturas vinculadas con la investigación. En la tabla 2 se muestra el cambio entre los dos programas.

\section{Tabla 2:}

Asignaturas relacionadas con la investigación educativa en los programas antiguos y renovados

\begin{tabular}{|c|c|}
\hline Asignaturas currículum antiguo & Asignaturas currículum renovado \\
\hline $\begin{array}{l}\text { Metodología del Diagnóstico } \\
\text { Educacional, (1er semestre) } \\
\text { Construcción de Conocimiento en } \\
\text { Educación, (4to semestre) } \\
\text { Metodología de la Investigación } \\
\text { Educacional, (7mo semestre) } \\
\text { Seminario de Licenciatura, (8vo } \\
\text { semestre) }\end{array}$ & $\begin{array}{l}\text { Teorías de la Educación, (4to semestre) } \\
\text { Epistemología y Paradigmas de la } \\
\text { Investigación Educativa, (5to semestre) } \\
\text { Análisis de Proyectos de Investigación } \\
\text { Educativa, (6to semestre) } \\
\text { Metodología de la Investigación } \\
\text { Educativa, (7mo semestre) } \\
\text { Seminario de Licenciatura en Educación, } \\
\text { (8vo semestre) }\end{array}$ \\
\hline
\end{tabular}

Fuente: Elaboración propia.

Se espera que este cambio curricular tenga consecuencias favorables en la formación pedagógica de los futuros profesores, que las modificaciones no queden sólo en "el papel" y que se comience a observar la investigación educativa como un elemento crucial en las carreras de pedagogía. La real repercusión de los cambios curriculares podrá ser analizada una vez que las generaciones que iniciaron sus estudios en el ańo 2019 finalicen su carrera e ingresen al mundo laboral. Sin embargo, resulta alentador observar en el perfil de egreso de los licenciados en educación en los programas renovados, por ejemplo:

Graduado/a universitario/a que posee conocimientos de la estructura conceptual y epistemológica de las ciencias de la educación, de los fenómenos y situaciones educativas, y conocimientos básicos de investigación educacional para analizar los problemas educativos y generar conocimientos profesionales asociados al mejoramiento de su propio desempeño, de la unidad educativa de pertenencia y de los contextos próximos (Decreto exento 290/2018, Pedagogía en Castellano y Filosofía, Universidad de La Serena, Chile, p.3).

\subsection{La importancia de que los formadores de profesores tengan un rol investigador}

El rol de docentes que dan clases en las pedagogías es fundamental para que la formación en investigación se optimice. Al respecto, y si bien Larenas, Rodríguez, Hernández, Solar y Morales (2015) no centran su estudio específicamente en futuros docentes, sí obtienen hallazgos relevantes sobre su formación en investigación. Ellos realizan un estudio que pretende analizar los temas claves en la formación de profesores desde las percepciones de profesores en la universidad y de profesores en ejercicio. En las preguntas sobre la formación inicial, un 65\% de los docentes universitarios plan- 
tea que enseña herramientas investigativas a sus estudiantes y un $28 \%$ señala que sí forma a futuros investigadores. Hubiera sido interesante que el estudio mencionara si los docentes universitarios participantes tienen o no el grado de doctor, tópico que también es una arista a considerar en este análisis. En el contexto chileno, es una realidad que no todos los formadores de profesores son investigadores ni tampoco cuentan necesariamente con el grado de doctor. Es cierto que actualmente las universidades se están sometiendo a criterios de evaluación más exigentes debido al rol fiscalizador de la Comisión Nacional de Acreditación (CNA), y que se está solicitando que los docentes tengan el grado de magíster o el de doctor.

Es importante indicar que tener el título de doctor no garantiza que un docente investigue. Claramente, hay doctores que no investigan como también hay profesionales que teniendo un magíster sí desarrollan una carrera investigativa. Sin embargo, es de un doctor del cual se espera que publique estudios en su área. No se pretende subestimar el grado de licenciado o de magíster, pero sí se intenta destacar que el grado de doctor, al ser el nivel universitario más alto, debería ser un requisito importante para quienes están formando profesores de calidad. Pero eso no es todo, ese doctor debería comprometerse con ejercer un rol investigador en su área.

Buscando ejemplos de contextos iberoamericanos que permitan ver otro enfoque del tema, destaca el caso de España, país que cuenta con la Agencia Nacional de Evaluación de la Calidad y Acreditación (ANECA). En el caso del profesorado universitario, la ANECA establece una serie de criterios para acreditar a un docente universitario y, por ende, para habilitarlo para trabajar en una institución pública. Si bien este modelo de evaluación ha recibido algunas críticas, lo cierto es que establece criterios unificados a nivel nacional que permiten seleccionar a quienes impartirán docencia universitaria, y luego organiza la escala de jerarquización universitaria.

Toda esta descripción permite contextualizar un elemento crucial para lograr la alfabetización de los futuros docentes: si un formador de profesores no es un investigador, ¿se interesará por incluir a la investigación educativa entre los lineamientos de su asignatura? ¿Hay una preocupación del formador sobre lo que realmente necesita el futuro profesor a nivel investigativo? Si el formador no está familiarizado con la investigación es difícil que se pueda incorporar en la formación de profesores, tanto a nivel instrumental como a nivel formativo.

En el primer caso, por ejemplo, los requisitos del Sistema de Desarrollo Profesional Docente Chileno (Ley 20.903) exigen que los futuros profesores tengan habilidades para investigar sobre su propia práctica educativa en el contexto escolar.

En el segundo caso, los futuros profesores deben apropiarse de las habilidades de investigación, en cuanto es un recurso para ser profesional de la educación, uno que toma decisiones, actúa por conocimiento y no es meramente un técnico de la enseñanza.

En este camino, el acompañamiento por parte de un docente universitario que le atribuye importancia a la investigación educativa es fundamental. Lograrlo no es siempre una tarea fácil. Ahora bien, también es cierto que los formadores de profesores necesitan el apoyo de las instituciones para continuar con estudios de posgrado y formarse como investigadores, lo que en algunos casos se convierte en otro problema a resolver. Las universidades deben proporcionar las condiciones necesarias para que sus académicos se sientan con la tranquilidad, tiempo y confianza para convertirse en magísteres y/o doctores en su área. 


\subsection{La utilización de los artículos de investigación como recurso pedagógico}

Existen pocas investigaciones que estudien las valoraciones u opiniones de los futuros docentes sobre los artículos de investigación, y menos aún respecto a la utilización didáctica o pedagógica de los mismos dentro de sus asignaturas. Si bien existen algunos trabajos que metodológicamente utilizan la lectura de artículos, estos están focalizados principalmente en profesores en ejercicio (Bartels, 2003; Murillo, Perines \& Lomba, 2017; Zeuli, 1994).

A pesar de la carencia de datos empíricos sobre el tema, resulta lógico sostener que el artículo de investigación puede ser utilizado como un recurso en el aula y que esta utilización puede impactar positivamente en la formación de profesores. El desafío es cómo se puede sistematizar este proceso. A continuación, una posible propuesta:

- Sensibilización de los estudiantes con los artículos de investigación a través de algunas sesiones centradas en sus objetivos y resultados.

- Contacto de los estudiantes con una serie de artículos centrados en distintos temas pedagógicos.

- Dinámicas grupales de lectura y reflexión sobre el contenido de los artículos.

- Dinámicas grupales centradas en la utilización práctica del contenido de los artículos para el futuro trabajo de un profesor.

En todos los puntos mencionados subyace la idea de que habrá "alguien" detrás de este proceso, ese "alguien" debe ser el profesor formador, que idealmente maneje las competencias relativas a la investigación y que, mejor aún, sea un docente-investigador, tal como se destacaba en el punto anterior.

Sin embargo, una "alfabetización" no acaba cuando un futuro profesor lee un estudio. Para que los futuros docentes lleguen a conocer de manera consciente las características y funciones de la investigación necesitan recibir información adecuada sobre ella (De la Orden \& Mafokozi, 1999). Lo que requieren es consultar información que puedan conectar con su propia experiencia profesional. Si las lecturas no guardan relación con el contexto en el que se ubicarán en el futuro, no tendrán sentido para ellos y desestimarán su valor (Tom, 1997).

\subsubsection{Estrategias de aprendizaje basadas en la investigación:}

La lectura y revisión de artículos de investigación en la formación de profesorado también encuentra fundamento en la metodología del Aprendizaje Basado en Investigación (ABI), definida como una estrategia didáctica activa centrada en el alumno, para desarrollar competencias que le permitan realizar una investigación (Peñaherrera, Chiluiza \& Ortiz, 2014). En el ABI se contempla la utilización sistemática y progresiva de la investigación en la formación universitaria con la orientación del docente. La indagación, el aprendizaje por descubrimiento y el aprendizaje colaborativo son algunas de sus prioridades. La Universidad de Warwick, en el Reino Unido, y el South Carolina Honors College han sido pioneras en la utilización intencionada del ABI entre sus prácticas y es de esperar que en Latinoamérica se comience a hablar más de ello. 


\subsection{La inclusión de estudiantes de pedagogía en proyectos de investigación}

Counsell, Evans, McIntyre y Raffan (2000) proponen algunos principios que resultan fundamentales para la utilización exitosa de la investigación en los docentes en formación. Uno de ellos es que los futuros profesores necesitan ayuda para aprender a participar en la investigación sobre su propia práctica.

En esta línea, una buena estrategia para alfabetizar a los futuros profesores puede ser su incorporación en proyectos de investigación donde tengan la oportunidad de colaborar con investigadores, profesores en ejercicio y otros actores involucrados. El esfuerzo para incluirlos debe proceder, por ejemplo, de parte de la administración pública que financia los proyectos de investigación (en el contexto chileno, hay algunos avances al respecto en los concursos ofrecidos por Fondef y Fondecyt). Otro ejemplo de la incorporación de distintos agentes educativos en proyectos de investigación son los IPA (Investigación Pedagógica Aplicada), iniciativa que contempla la realización de una investigación "situada" en una escuela y que requiere de la participación de estudiantes de pedagogía junto a investigadores y profesores del sistema escolar.

Otra manera de lograr esta implicación es a través de los semilleros de investigación, los cuales, de acuerdo con Hernández-Pino (2005), son grupos disciplinares o interdisciplinares autónomos donde estudiantes universitarios desarrollan estudios con el propósito de iniciar tempranamente y/o fortalecer su formación en investigación. En esta misma línea, Aldana (2010) define los semilleros de investigación como grupos líderes para formar el espíritu investigativo. Sobre ello, es valioso el relato del profesor colombiano Jorge Ossa, para quien los semilleros se han convertido en una de las experiencias pedagógicas más enriquecedoras de su carrera:

(...) en la década de los 90s, ocurrió que mi laboratorio se fue llenando de estudiantes de pregrado que llegaron atraídos por los proyectos (...) más allá de la actividad técnica específica, no había un espacio para la reflexión crítica y para el diálogo que permitiera poner en clave de formación toda esta actividad febril de "investigación”. Ante esta situación surgió la invitación que se materializó en lo que los mismos muchachos bautizaron como Semilleros de Investigación (Ossa, p.5).

Instaurar y potenciar los semilleros es una forma concreta de conectar el trabajo de los investigadores con los intereses pedagógicos de los futuros profesores. Sería ideal que las iniciativas particulares de semilleros dentro de las universidades se fueran institucionalizando hasta convertirse en programas nacionales reconocidos por el Ministerio de Educación, tal como sucede, por ejemplo, en la Universidad Católica de Colombia, que cuenta actualmente con 64 semilleros de investigación de distintas disciplinas, iniciativa ejemplar para las políticas institucionales de otros países iberoamericanos.

A propósito de ello, Colombia es uno de los países que pueden servir como referente de este tema a nivel iberoamericano, sin desconocer que la Universidad de Humboldt en Alemania es un enorme referente en la formación investigativa de los jóvenes universitarios a nivel internacional. En el contexto colombiano se ha promovido la formación de semilleros de investigación, con el objetivo de generar una masa crítica capaz de resolver la necesidad de contar con más y mejores investigadores (Quintero, Munévar \& Munévar, 2008). Los estudios realizados en dicho país reflejan resultados 
optimistas respecto a la participación de los estudiantes en eventos científicos, en instancias donde se socializan artículos científicos, ponencias y proyectos. También se destaca que las universidades están facilitando estos espacios y apoyando las iniciativas al respecto (Correa, 2006).

Saavedra-Cantor, Muñoz-Sánchez, Antolínez-Figueroa, Rubiano-Mesa y Puerto-Guerrero (2015) realizan un estudio donde revisan los avances en esta materia en Colombia y a partir de él mencionan algunos desafíos que se deben enfrentar, entre ellos, definir una política de semilleros estudiantiles que conciba la articulación de estos con el plan de estudios, garantizando de esta manera su permanencia y desarrollo. Los autores concluyen que los semilleros de investigación se caracterizan por ser contextos de discusión académica e investigativa que fortalecen las habilidades investigativas de estudiantes y docentes. "En ellos convergen distintas posturas filosóficas y formas de abordar los fenómenos, las cuales facilitan encontrar soluciones a las problemáticas desde la diversidad" (p.402).

\section{Conclusiones}

Si algo ha quedado claro es que la "alfabetización" de los futuros profesores necesita que estos desarrollen un habitus investigativo (Catalán, 2017). Desarrollar este habitus no es tarea fácil porque requiere de una transformación profunda en los lineamientos de los planes de estudio. Muchas veces los cambios no son bienvenidos en las instituciones de tradición (y está claro que muchas universidades lo son), por lo que estas iniciativas chocan con la realidad burocrática de los procesos. La investigación necesita ser incorporada de manera transversal en el currículo, integrada como resultado del aprendizaje, y no debe formar parte de un par de asignaturas prácticamente desconectadas del resto del programa. Los futuros profesores deberían tener la oportunidad de participar, por ejemplo, en seminarios de investigación, en lectura de artículos de investigación concernientes a su futura práctica, en la observación de realidades pedagógicas desde las cuales extraer problemas de investigación, en la elaboración de micro-estudios vinculados con la comunidad local, etc. Además, los docentes en formación deben adquirir las competencias necesarias para comprender el conocimiento generado por la investigación, lo que no es tan simple como parece. Comprender el "idioma" de la investigación exige a los docentes tener mayores experiencias y competencias (Gore \& Gitlin, 2004). Manejar este lenguaje requiere de un proceso gradual y organizado que respete una progresión cognitiva que se debe reflejar en los resultados del aprendizaje que componen los planes de estudio.

Lograr alfabetizar a los futuros docentes implica la compleja tarea de comprender que como educadores no solo deben ser receptores o consumidores de conocimiento, sino que también deben participar en producirlo (Catalán, 2017).

Si los estudiantes logran implicarse con la investigación de manera responsable, informada y persistente en el tiempo pueden ampliar sus puntos de vista en la búsqueda de mejores soluciones a los problemas de su práctica (Elliot, 2001). El Aprendizaje Basado en Investigación, los IPA y los semilleros de investigación son algunas ideas que pueden servir de base.

Las modificaciones en la formación inicial son imprescindibles para cambiar la cultura del profesorado, logrando que sientan su profesión de una manera más cercana a la producción del conocimiento pedagógico y más vinculada a la actualización y reflexión de los procesos educativos involucrados en dicha producción. 


\section{Referencias}

Aldana, L. (2010). Creando semilleros de investigación en la escuela. Gondola, Enseñanza y Aprendizaje de las Ciencias, 5(1), 3-10.

Anwaruddin, S. (2016). Language teachers' responses to educational research: Addressing the 'crisis' of representation. International Journal of Research \& Method in Education, 39(3), 314-328. https://doi.org/10.1080/1743727X.2016.1166485

Anwaruddin, S., \& Pervin, N. (2015). English-language teachers' engagement with research: Findings from Bangladesh. Professional Development in Education, 41(1), 21-39. https://doi.org/10.1080/19415257.2013.861860

Bartels, N. (2003). How teachers and researchers read academic articles. Teaching and Teacher Education, 19(7), 737-753. https://doi.org/10.1016/j.tate.2003.06.001

Burn, K. (2007). Professional knowledge and identity in a contested discipline: Challenges for student teachers and teacher educators. Oxford Review of Education, 33(4), 445-467. https://doi.org/10.1080/03054980701450886

Catalán, J. (2017). Investigación y Docencia: Una vinculación necesaria y posible. En F. Araya (Ed.), Desarrollo del Pensamiento Geográfico: Aportes para la vinculación entre Investigación Docencia (17-32). La Serena: Editorial Universidad de La Serena.

Comisión Nacional de Acreditación. Criterios de evaluación de carreras de educación. Santiago de Chile. Recuperado de https://bit.ly/2hocrpq

Correa, L. (2006). Estado actual del trabajo en red de los semilleros de investigación en Colombia. Revista Opinión Jurídica, 5(10), 197-201.

Counsell, C., Evans, M., McIntyre, D., \& Raffan, J. (2000). The usefullness of educational research for trainee teachers' learning. Oxford Review of Education, 26(3-4), 467-482. https://doi.org/10.1080/713688548

CPEIP (2012). Estándares Orientadores para egresados de carreras de Pedagogía en Educación Básica. Santiago: LOM.

De la Orden, A., \& Mafokozi, J. (1999). La investigación educativa: Naturaleza, funciones y ambigüedad de sus relaciones con la práctica y la política educativas. Revista de Investigación Educativa, 17(1), 7-29.

Gore, J., \& Gitlin, A. D. (2004). [Re] Visioning the academic-teacher divide: Power and knowledge in the educational community. Teachers and teaching, 10(1), 35-58. https://doi.org/10.1080/13540600320000170918

Grossman, P., \& McDonald, M. (2008). Back to the future: Directions for research in teaching and teacher education. American Educational Research Journal, 45(1), 184-205. https://doi.org/10.3102/0002831207312906

Haberfellner, C., \& Fenzl, T. (2017). The utility value of research evidence for educational practice from the perspective of preservice student teachers in Austria - A qualitative exploratory study. Journal for Educational Research Online, 9(2), 69-87.

Hall, E., Leat, D., Wall, K., Higgins, S., \& Edwards, G. (2006). Learning to lern: Teacher research in the zone of proximal development. Teacher Development, 10(2), 149-166.

https://doi.org/10.1080/13664530600773119

Harris, D., \& Sass, T. R. (2011). Teacher training, teacher quality and student achievement. Journal 
of Public Economics, 95(7-8), 798-812. https://doi.org/10.1016/j.jpubeco.2010.11.009

Hernández-Pino, U. (2005). Propuesta curricular para la consolidación de los semilleros de investigación como espacios de formación temprana en investigación. Revista Electrónica de la Red de Investigación Educativa, 1(2), 1-13.

Kaestle, C. (1993). The awful reputation of education research. Educational Researcher, 22(1), 2331. https://doi.org/10.2307/1177303

Lampert, M. (2009). Learning teaching in, from, and for practice: What do we mean? Journal of Teacher Education 61(1-2), 21-34. https://doi.org/10.1177/0022487109347321

Larenas, C. H. D., Rodríguez, M. I. S., Hernández, V. S., Solar, M. C., \& Morales, J. V. (2015). Temas clave en la formación de profesores en Chile desde la perspectiva de docentes y directivos. Revista Complutense de Educación, 26(3), 543-569.

Murillo, F., \& Perines H. (2017). Cómo los docentes no universitarios perciben la investigación educativa. Revista Complutense de Educación, 28(1), 81-99. https://doi.org/10.5209/rev_RCED.2017.v28.n1.48800

Murillo, F., Perines H., \& Lomba, L. (2017). La comunicación de la investigación educativa. Una aproximación a la relación entre la investigación, su difusión y la práctica docente. Profesorado, Revista de Curriculum y Formación del Profesorado, 21(2), 182-200.

Ossa, J. (2005). Educar es enseñar a indagar la investigación como proceso de formación. Educaçao, 28(3), 525-533.

Pekel, F. O., \& Akçay, S. (2018). Are science teachers really aware of the importance of educational research? European Journal of Education Studies, 4(3), 196-214.

Peñaherrera, M., Chiluiza, K. y Ortiz, A. (2014). Inclusión del aprendizaje basado en investigación (ABI) como práctica pedagógica en el diseño de programas de postgrados en Ecuador. Elaboración de una propuesta. Journal for Educators, Teachers and Trainers, 5(2), 204-220. Recuperado de https://bit.ly/2M45hYR

Perines H., \& Murillo F. (2017a). Percepciones de los docentes en formación sobre la investigación educativa. Estudios Pedagógicos, 43(1), 251- 268. https://doi.org/10.4067/S0718-07052017000100015

Perines, H., \& Murillo F. (2017b). ¿Cómo mejorar la investigación educativa? Sugerencias de los docentes. Revista de la Educación Superior, 46(181), 89-104. https://doi.org/10.1016/j.resu.2016.11.003

Perines, H. (2016). Las difíciles relaciones entre la investigación educativa y la práctica docente (Tesis doctoral). Universidad Autónoma de Madrid, Madrid.

Procter, R. (2013). Teachers and research: What they value and what they do. Journal of Pedagogic Development, 3(1), 31-37.

Quintero, J., Munévar, R. A. \& Munévar, F. I. (2008). Semilleros de investigación: Una estrategia para la formación de investigadores. Educación y Educadores 11(1), 31-42.

Rubio, M. J., Torrado, M., Quirós, C., \& Valls, R. (2018). Autopercepción de las competencias investigativas en estudiantes de último curso de Pedagogía de la Universidad de Barcelona para desarrollar su trabajo de fin de grado. Revista Complutense de Educación, 29(2), 335-354.

Rust, F. (2009). Teacher research and the problem of practice. The Teachers College Record, 111(8), 1882-1893.

Saavedra-Cantor, C. J., Muñoz-Sánchez, A. I., Antolínez-Figueroa, C., Rubiano-Mesa, Y. L., \& 
Puerto-Guerrero, A. H. (2015). Semilleros de investigación: Desarrollos y desafíos para la formación en pregrado. Educación y Educadores, 18(3), 391-407.

Shkedi, A. (1998). Teachers' attitudes towards research: a challenge for qualitative researchers. International Journal of Qualitative Studies in Education, 11(4), 559-77. https://doi. org/10.1080/095183998236467

Simons, H., Kushner, S., Jones, K., \& James, D. (2003). From evidence-based practice to practice based evidence: The idea of situated generalization. Research Papers in Education, 18(4), 347364. https://doi.org/10.1080/0267152032000176855

Slavin, R. (2002). Evidence-based education policies: Transforming educational practice and research. Educational Researcher, 31(7), 15-21.

Scherping, G. (2010). El caso de la formación de profesores. En Consejo Nacional de Educación/ Comisión Nacional de Acreditación. Seminario Internacional 2009. Calidad de los egresados, responsabilidad social ineludible. (pp. 175-178). Santiago de Chile: Andros Impresores.

Stenhouse, L. (1987). La investigación como base de la enseñanza. Madrid: Morata.

Taylor, M. (2013). Social science teachers' utilisation of best evidence synthesis research. New Zealand Journal of Educational Studies, 48(2), 34-50.

Tom, A. (1997). Redesigning teacher education. Albany, NY: State University of New York Press.

Vanderlinde, R., \& Van Braak, J. (2010). The gap between educational research and practice: Views of teachers, school leaders, intermediaries and researchers. British Educational Research Journal, 36(2), 299-316. https://doi.org/10.1080/01411920902919257

Zeuli, J. S. (1994). How do teachers understand research when they read it? Teaching and Teacher Education, 10(1), 39-55. https://doi.org/10.1016/0742-051X(94)90039-6 